\title{
Advanced Optimization of Hybrid-Electric-Vehicle Drivelines using Engine-in-the-Loop Simulation
}

\author{
Benjamin Tilch*, Thorsten Reimers, Peter Eilts
}

Technische Univ. Braunschweig, Niedersächsisches Forschungszentrum Fahrzeugtechnik (NFF), Inst. of Internal Combustion Engines Braunschweig (ivb), Langer Kamp 6, 38106 Braunschweig, Germany; *b.tilch@tu-bs.de

Abstract. At the Institute of Internal Combustion Engines of Technische Universität Braunschweig (ivb) a modular real-time longitudinal vehicle simulation model has been developed. The main goal of the vehicle model is the calculation of boundary conditions for the virtual development process of internal combustion engines (ICE). Especially in hybrid electric vehicle (HEV) development an efficient tool is necessary to solve the nearly unmanageable task to find the optimum configuration of hybrid topology, degree of hybridization and efficient operation strategy. To achieve optimal results several ICE models with different levels of detail, depending on the state of development, were used.

By coupling a real-time vehicle model and a hardware-inthe-loop ( $\mathrm{HiL}$ ) test bench engine characteristics like fuel consumption and exhaust gas emissions can be analysed.

The use of engine-in-the-loop (EiL) provides the opportunity to integrate hard to simulate parameters in hybrid strategies. By using identical vehicle models for the power train design process and experimental studies at the test bench systematic errors can be avoided. Additionally the development time decreases. Furthermore the EiL method enables the development of any kind of hybrid power train and helps to design intelligent and unconventional hybrid strategies online.

\section{Introduction}

Increasing oil prices, limited fossil resources and growing environmental requirements are a huge challenge in a world of rising demand for individual mobility. The aim of reducing fossil energy consumption and the greenhouse gas emission production demands alternative, innovative and efficient power train solutions. Beside the optimization of ICE's efficiency the combination with an electric motor in an HEV is a possibility to reach these targets.

Between conventional and electric vehicles almost all interim solutions are possible by combining ICE, EM and battery in hybrid structures as well as different degrees of hybridization. Figure 1 shows conceivable types of HEV power trains. To find the optimal combination in this parameter space an intelligent engineering method is obligatory. Because of the wide range of solutions a strategic method must be used to handle the almost unmanageable task of finding an optimal HEV configuration in minimum development time.

Based on the preliminary studies at the ivb [1], [2] a combined method of real-time simulation and experimental engine testing has been developed to manage this challenge.

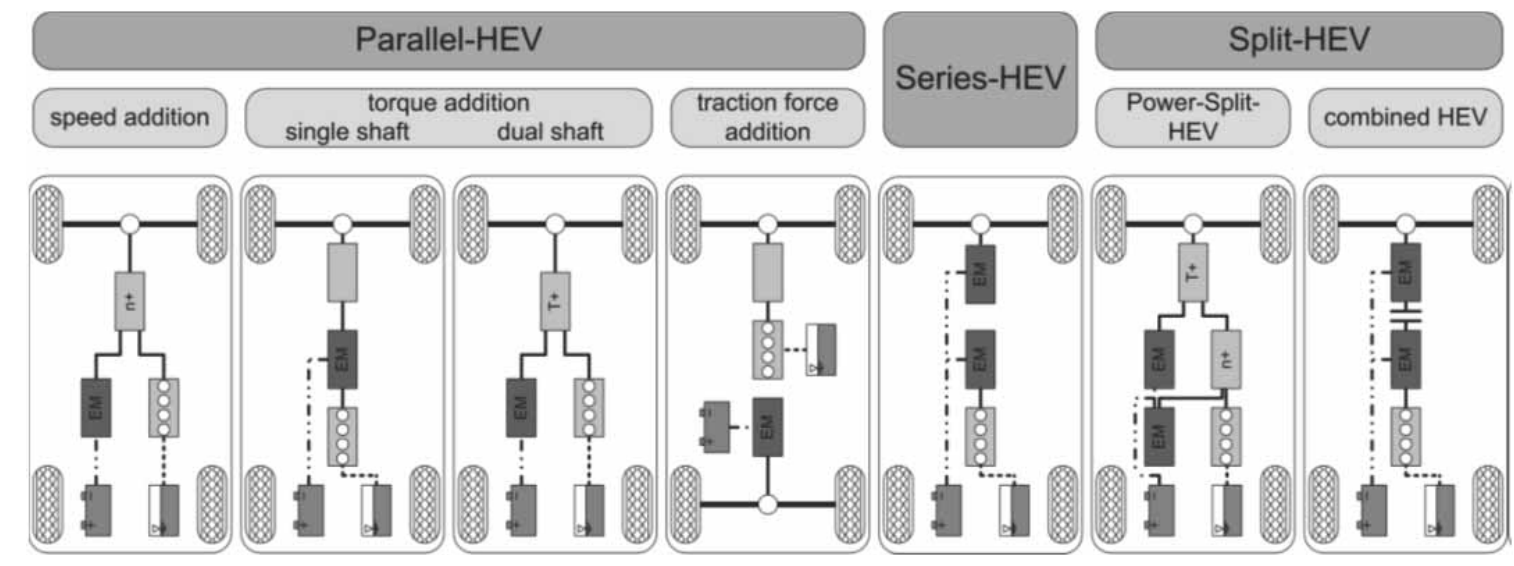

Figure 1. Overview of HEV power trains. 


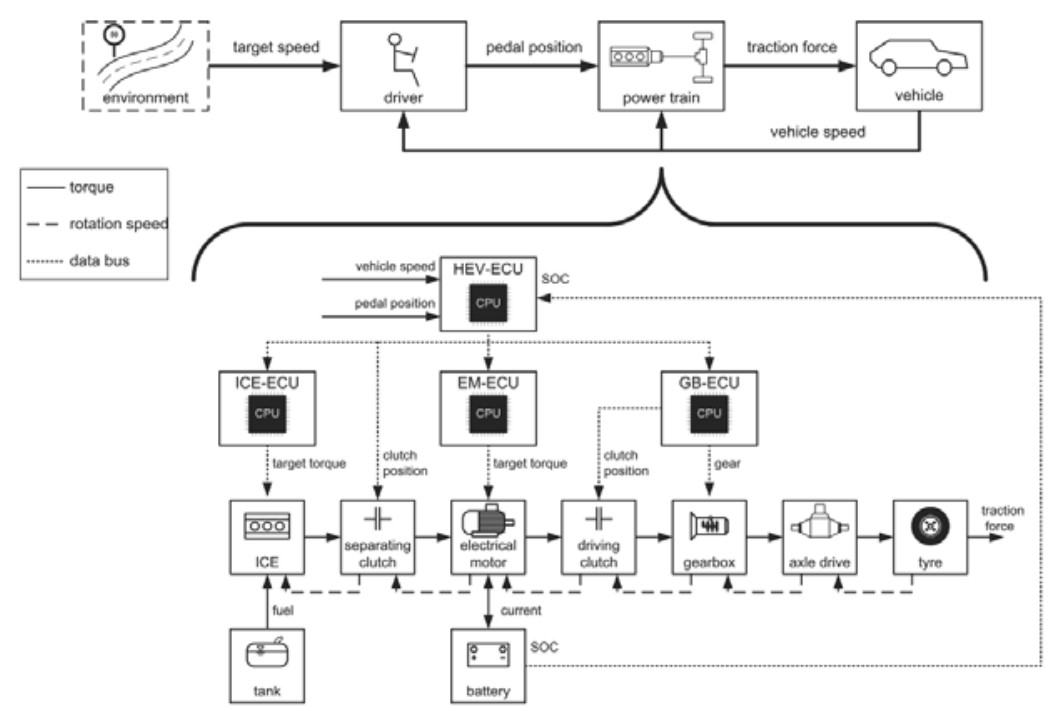

Figure 2. Configuration of the longitudinal HEV-model with parallel power train structure.

Besides different ICE models with different levels of detail a co-simulation with an engine test bench is possible. By replacing the simulated ICE by hardware at the EiL test bench research on the real engine behaviour for example considering thermal influences in engine friction or exhaust gas emissions can be done.

\section{Vehicle Simulation Model and Engine-in-the-Loop test bench}

\subsection{Vehicle Simulation Model}

For the experimental studies a longitudinal HEV drive train was modelled in Matlab/Simulink. An overview of the simulation model for a parallel HEV gives Figure 2.

The model is built up as a forward-looking-model and is divided in driver-, power train and vehicle model. In addition to the physical models of ICE, electrical motor (EM), battery, gearbox and axle drive, electronic control units (ECU) for ICE, EM and gearbox are necessary. Supervisory an HEV operating ECU manages the interaction of the other component-ECUs. The HEV-ECU splits the target torque between the ICE and the EM depending on the operation strategy. Furthermore the HEV-ECU triggers the two clutches between ICE and EM and between EM and gearbox.

The driver model acts as a PID controller. Due to comparison the current vehicle speed and the target speed the driver actuates the accelerator or brake pedal based on the control deviation.
In the power train model the traction force is calculated from the accelerator position. The power train model depends on the HEV structure and considers the losses, inertias and transmissions.

A tyre model transforms the brake pedal position into a brake force. In the vehicle model the available traction or brake force is balanced with the vehicle driving resistances.

In the model vehicle the longitudinal forces are balanced (Eq. 1). The resulting traction force depends on the driving resistances: rolling, air, gradient and acceleration resistant (Eqs. 2-6).

Traction force:

$$
\begin{array}{r}
Z=\sum_{j=1}^{n} \frac{T_{t j}}{r_{j}}=F_{\text {rol }}+F_{\text {air }}+F_{\text {gra }}+ \\
F_{a c c}
\end{array}
$$

Rolling resistance:

$$
\begin{aligned}
& F_{r o l}=m_{v e h} \cdot g \cdot f_{r} \\
& f_{r}=f_{r, 0}+f_{r, 1} \cdot x_{v e h}+f_{r, 4} \cdot x_{v e h}{ }^{4}
\end{aligned}
$$

Air resistance:

$$
F_{\text {air }}=c_{w} \cdot A \cdot \frac{\varrho}{2} \cdot \dot{x}_{v e h}^{2}
$$

Gradient restistance:

$$
F_{g r a}=m_{v e h} \cdot g \cdot \sin \alpha
$$

Acceleration resistance:

$$
F_{a c c}=\lambda \cdot m_{v e h} \cdot \ddot{x}_{v e h}
$$

In consideration of the vehicle speed the driving power can be calculated (Eq. 7).

Driving power:

$$
P_{v e h}=Z \cdot \dot{x}_{v e h}=\left(F_{r o l}+F_{a i r}+F_{g r a}+F_{a c c}\right) \cdot x_{v e h}
$$

In the simulation model the traction force is totalled by the drive torques of ICE and EM divided by the dynamic tyre rolling radius. By converting Eq. 1 according to the unknown acceleration force and in consideration of the break force Fbra from the tyre model the vehicle acceleration can be calculated (Eq. 8). The integration of the vehicle acceleration delivers the actual vehicle speed (Eq. 9). Integrating Eq. 9 results in driving distance (Eq. 10). 
Vehicle acceleration:

$$
\ddot{x}_{v e h}=\frac{F_{a c c}}{m_{v e h}}=\frac{Z-F_{r o l}-F_{a i r}-F_{g r a}-F_{b r a}}{m_{v e h}}
$$

Vehicle speed:

$$
\dot{x}_{v e h}=\int \ddot{x}_{v e h} d t
$$

Vehicle driving distance: $\quad x_{v e h}=\int \dot{x}_{v e h} d t$

Between ICE and EM a separating clutch is installed to realize electrical driving. A second clutch between EM and gearbox allows disconnection of ICE/EM and power train. This clutch allows battery charging during vehicle standstill.

The gearbox can be simulated as a simple or as a dual clutch transmission. In case of the dual clutch transmission the separate driving clutch is not required. The dual clutch transmission is divided physically in two partial transmissions with one clutch each.

Depending on the pedal position and the vehicle speed the gears of the partial transmissions are prechosen by the gearbox-ECU (GB-ECU). At a shifting event one clutch opens while the other clutch closes simultaneously. As a result the gear changes almost without traction interruption. The gearbox losses are interpolated from maps as a function of gear, speed and torque. In addition the torque flow direction is considered.

This is necessary to account for the difference between driven and non-driven (recuperation) operation mode. The EM efficiency is modelled by maps as a function of the operation point, which are divided in motor mode and generator mode. The input of the battery model is given by the supplied or removed EM electrical power. Additionally the charging and discharging efficiencies are considered. The stored energy of the battery is calculated by integration of the charging and discharging power. One of the most important inputs for the HEVECU for choosing an energy optimal operating mode is the battery state of charge (SOC). It is calculated from the quotient of actual energy content and nominal energy content.

\section{Charging power:}

$$
P_{\text {bat, charge }}=\eta_{\text {charge }} . P_{\text {el,gen }}
$$

Discharging power:

$$
P_{\text {bat }, \text { charge }}=\frac{1}{\eta_{\text {discharge }}} \cdot P_{\text {el,mont }}
$$

Energy content:

$$
E_{b a t}=\int_{t_{1}}^{t_{2}} P_{b a t} d t
$$

State of charge:

$$
S O C=\frac{E_{\text {bat }}}{E_{\text {bat,nom }}}
$$

For the ICE three models with different levels of detail are integrated. Simple longitudinal design studies can be done with a map-based ICE model. In this simplest model the current ICE torque is calculated depending on the input target torque. To model the ICE transient behaviour, the inertia and the turbocharger response must be considered by correction functions which are calibrated by test bench measurements. The operation map limits for full load, friction curve, idle and maximum speed are considered by characteristic curves. Figure 3 shows characteristic operation maps of ICE and EM in a parallel HEV power train.

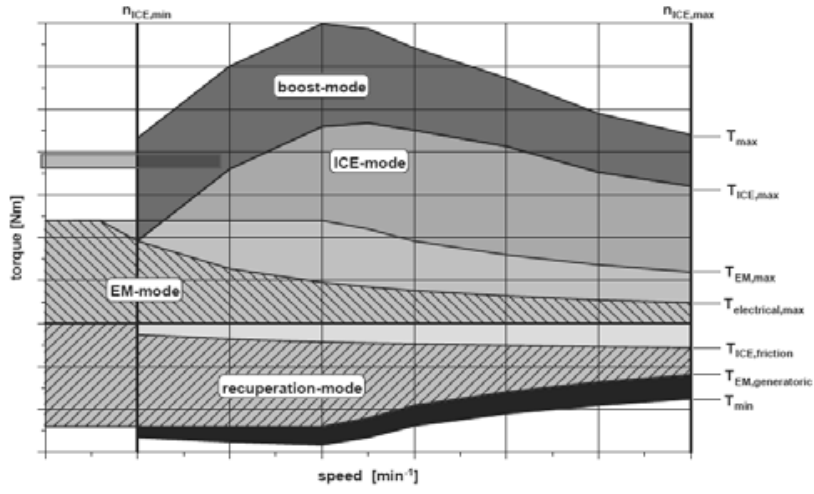

Figure 3. Operation maps of ICE and EM in a parallel HEV.

To simulate the transient thermal behaviour of the ICE, a thermal model can be used. The engine structure is build up as a lumped capacity model based on [3]. In this model the engine is divided in 18 capacities which interact via heat transfer network.

Furthermore a friction model has been developed which considers engine speed, coolant temperature, oil temperature and engine load depending on the ICE assemblies crankshaft, camshaft, piston and auxiliaries. The thermal losses of the combustion chamber are modelled as maps which are based on in-cylinder pressure analysis measured at an engine test bench. Simple models are used for the coolant and oil circuit.

For detailed analysis of the combustion process a complex thermodynamic model can be used which considers the gas dynamics in the inlet and outlet pipes. This model was set up with the software GT Power from Gamma Technologies and allows studying transient thermal effects of the combustion process like cooling down of the engine structure during stop phases. 
For this purpose a simplified 3d-model of the engine structure allows detailed analysis of the thermal losses in the combustion chamber. In addition the model gives realistic information about the ICE fuel consumption. A detailed ICE-ECU is necessary for the combustion model to control fuel injection, turbo-charging and exhaust gas recirculation.

To verify the simulation results, the ICE can be replaced by real hardware at the EiL test bench. The residual vehicle model runs in real time on the automation system.

Parameters that are difficult to simulate like ICE exhaust gas emissions can be measured directly. This allows the evaluation of the exhaust gas emissions which depend on the operation mode considering the ICE efficiency. In this way the benefit of different operating modes can be rated under realistic conditions likewise. Figure 4 shows an overview of the ICE models. With proceeding availability different models like EM can be replaced by hardware at a power train test bench.

Superior to all components with specific ECUs the HEV-ECU manages the vehicle operation mode and the corresponding torque split between ICE and EM. The HEV-ECU first indentifies the required driving power due to the vehicle speed and pedal positions. Based on this input parameters the HEV-ECU chooses from following operating modes (Fig. 3):

- Electrical driving: The EM delivers the necessary driving power while the ICE is shut off and the separating clutch is open. Fig. 3 clarifies that not the complete available EM power is useable for electrical driving. A safety range is necessary to get over the friction and inertia losses at the next ICE start procedure.

- ICE start: If the required driving power exceeds the electrical driving limit, the ICE has to start. The EM speeds up the ICE to actual power train speed.

- Boost mode: In boost mode the EM assists the ICE at transient conditions. For example the boost mode can improve the vehicle acceleration or mooth the transient ICE load. For this mode a high SOC is necessary.
- ICE Load Increasing: At low SOC the EM can be operated in generator mode to charge the battery. In this case the ICE load exceeds the required torque. This implies that the ICE works in a better specific fuel consumption operating point (Fig. 5). This operating mode requires a low SOC to avoid exceeding maximum battery energy content.

- Recuperation: In deceleration phases the EM can recuperate electrical energy from the kinetic energy of the vehicle. In this case the separating clutch opens to minimize ICE friction losses. Simultaneously the ICE is shut off.

- ICE single operation mode: This mode is equal to conventional vehicles. The prerequisite for this mode is that the required power exceeds maximum electrical driving power and SOC limitation so that no ICE load increasing is necessary.

- Battery loading at standstill: If the SOC drops below a critical limit the ICE can charge the battery at standstill phases. The separating clutch is closed while the driving clutch is open. The EM acts as a generator.

In addition special operating modes like smoothing transient engine load or limiting the maximum ICE torque, which are presented in [2], can be implied easily due to the modular model structure.

Figure 5 shows different operating strategies depending on the HEV power train structure compared to a conventional vehicle. The maps show the operating areas during New European Driving Cycle (NEDC). In a parallel HEV structure only load shifting is possible. A power split HEV allows torque and speed shifting of ICE operating point.

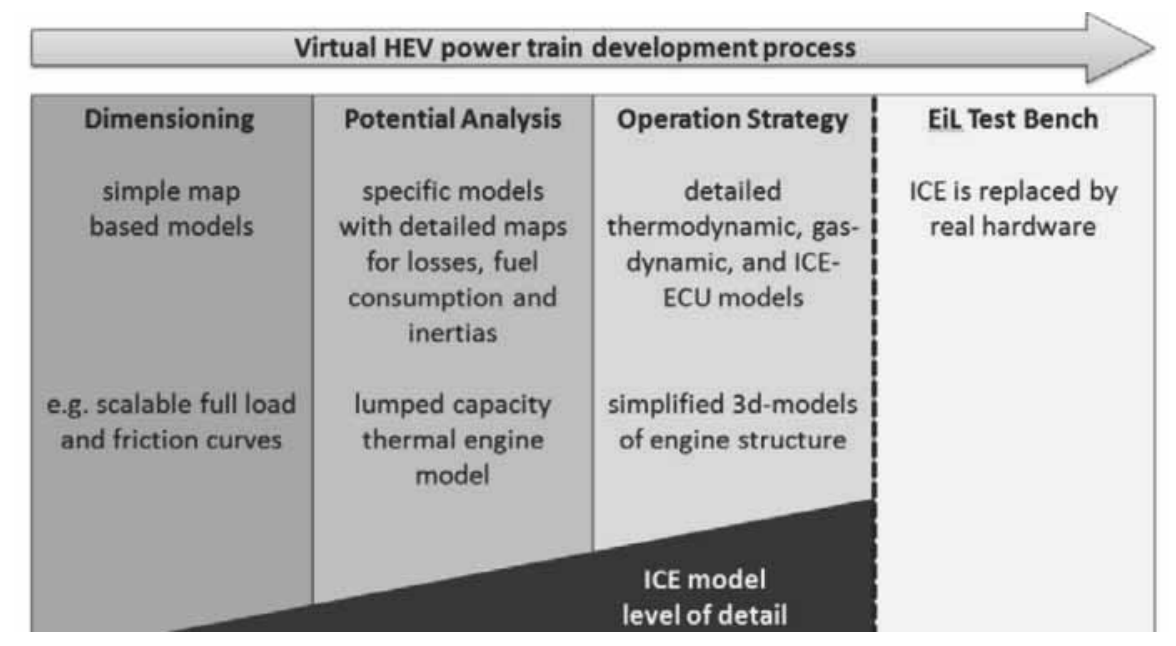

Figure 4. Available ICE models with different level of detail. 


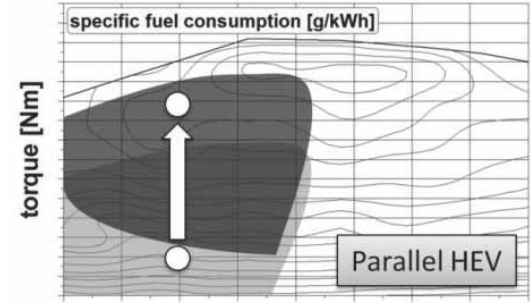

speed [min-1]

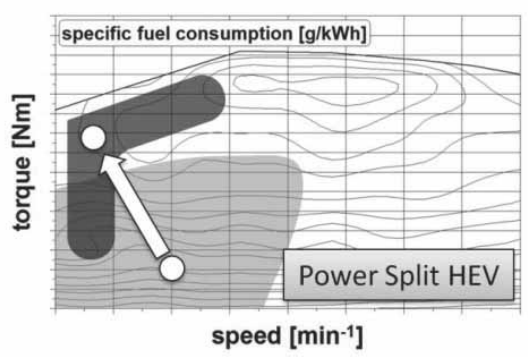

Figure 5. Operating point shifting depending on the HEV power train structure.

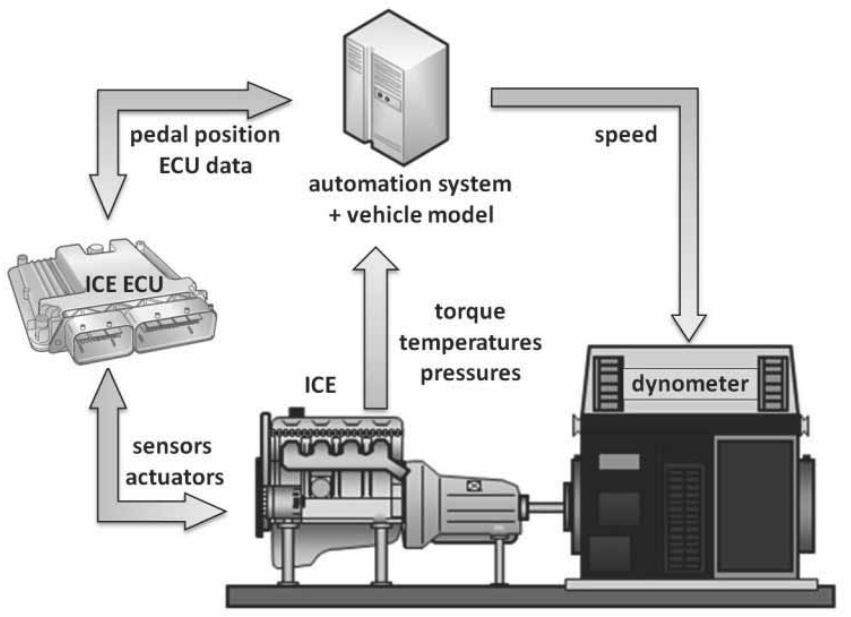

Figure 6. Engine-in-the-Loop test bench.

acceleration. ECU variables can be changed online by the simulation model after coupling the ICE control unit with the automation system. The ICE control unit communicates with the ICE by sensors and actuators. To consider ICE operation values like temperatures and pressures in HEV operation strategy the measured data are available in the test system. All measured and simulated data are recorded with up to $1 \mathrm{kHz}$.

\section{Simulation results}

Figure 7 shows test bench results of a co-simulation of a parallel HEV power train. In this example the ICE data are measured on real hardware while the electrical components and a dual clutch transmission are simulated. The figure shows the extra-urban part of the NEDC. In the first seconds the vehicle accelerates from standstill to a constant vehicle speed of $70 \mathrm{kph}$. By exceeding the electrical power limitation the ICE begins to operate.

\subsection{Engine-in-the-Loop Test Bench}

At the engine test bench the virtual ICE is replaced by real hardware. The remaining submodels like EM, battery or gearbox are simulated in real time directly at the test bench control PC. Compared to other cosimulation real-time systems, this solution generates the shortest cycle times. This procedure ensures that the real-time models which are used in the experimental setup are identical to the models in the early virtual development process.

For the experimental studies a turbocharged gasoline engine is used. The ICE is coupled to a dynamometer which is a highly dynamic asynchronous load machine with a maximum speed of $10.000 \mathrm{rpm}$ and a maximum torque of $450 \mathrm{Nm}$. Figure 6 gives an overview of the test bench configuration. The automation system controls the dynamometer and auxiliary systems like air and fluid conditioning. A test bench controller regulates both the dynamometer speed and the accelerator pedal position. Target values are calculated by the simulation model. The test bench sensors measure the ICE torque which is the input for the model to calculate the vehicle
To reach the target speed the GB-ECU shifts up to sixth gear. After reaching the target speed the vehicle is driven by the ICE. The GB-ECU now shifts up because of a decrease in the accelerator pedal position. Then the target speed decreases to $50 \mathrm{kph}$. Because of the constant power requirement and high SOC the ICE is shut off. The electrical power is sufficient for electrical driving. An obvious decrease of SOC is observable. In the following acceleration the power demand exceeds the limit of electrical driving which necessitates the ICE's restart. While the ICE starts a peak in EM torque can be seen. The high EM torque results from the EM generating driving power and power for the ICE start procedure at the same time.

The following acceleration phase is passed in ICE mode until the HEV-ECU shifts the operation mode to increasing ICE load in order to charge the battery. Compared to the first constant speed phase of $70 \mathrm{kph}$, where no ICE load increase is used, the SOC decreased below the limit of $50 \%$. Beyond this limitation the operation mode ICE load increase is enabled. To avoid a direct engine stop after exceeding this limitation a hysteresis is considered. 
During ICE load increasing the EM operates in generator mode to reach a higher ICE load compared to the required torque. This generates a benefit in specific ICE efficiency and a simultaneously increasing SOC. At the end of the driving cycle the vehicle decelerates from $120 \mathrm{kph}$ to standstill. While deceleration the EM works in generator mode and recuperates electrical energy from kinetic energy. To maximize the storable energy the ICE is shut off and the separating clutch opens to avoid ICE friction losses. This lifts up generator torque at the EM.

\section{Conclusion}

At the ivb a real-time HEV power train simulation model has been developed and adapted for co-simulation use at an EiL test bench. Depending on the development process models of different levels of detail are used. The EiL test bench offers the possibility that HEVs with different structures, degrees of hybridization and operation strategies can be analysed under realistic boundary conditions. This way parameters like exhaust gas emissions can be considered, that are difficult to simulate. The HEV-ECU can use both ICE parameters to optimize the operating strategy with focus on minimum fuel consumption and can adapt ICE-ECU parameters online. In future work the development method can be used as an efficient tool to evaluate different $\mathrm{HEV}$ power train solutions with an ICE within shortest time.

\section{References}

[1] Lindenkamp, N. (2011). Strategien zur Reduzierung der NOX- und Partikelemissionen eines Dieselhybridfahrzeugs Dissertation, Technische Universität Braunschweig, Sierke Verlag, 2011

[2] Lindenkamp, N.; Stöber-Schmidt, C.-P. and Eilts, P. (2009). Strategies for Reducing NOx- and Particulate Matter Emissions in Diesel Hybrid Electric Vehicles. SAE-paper 2009-01-1305.
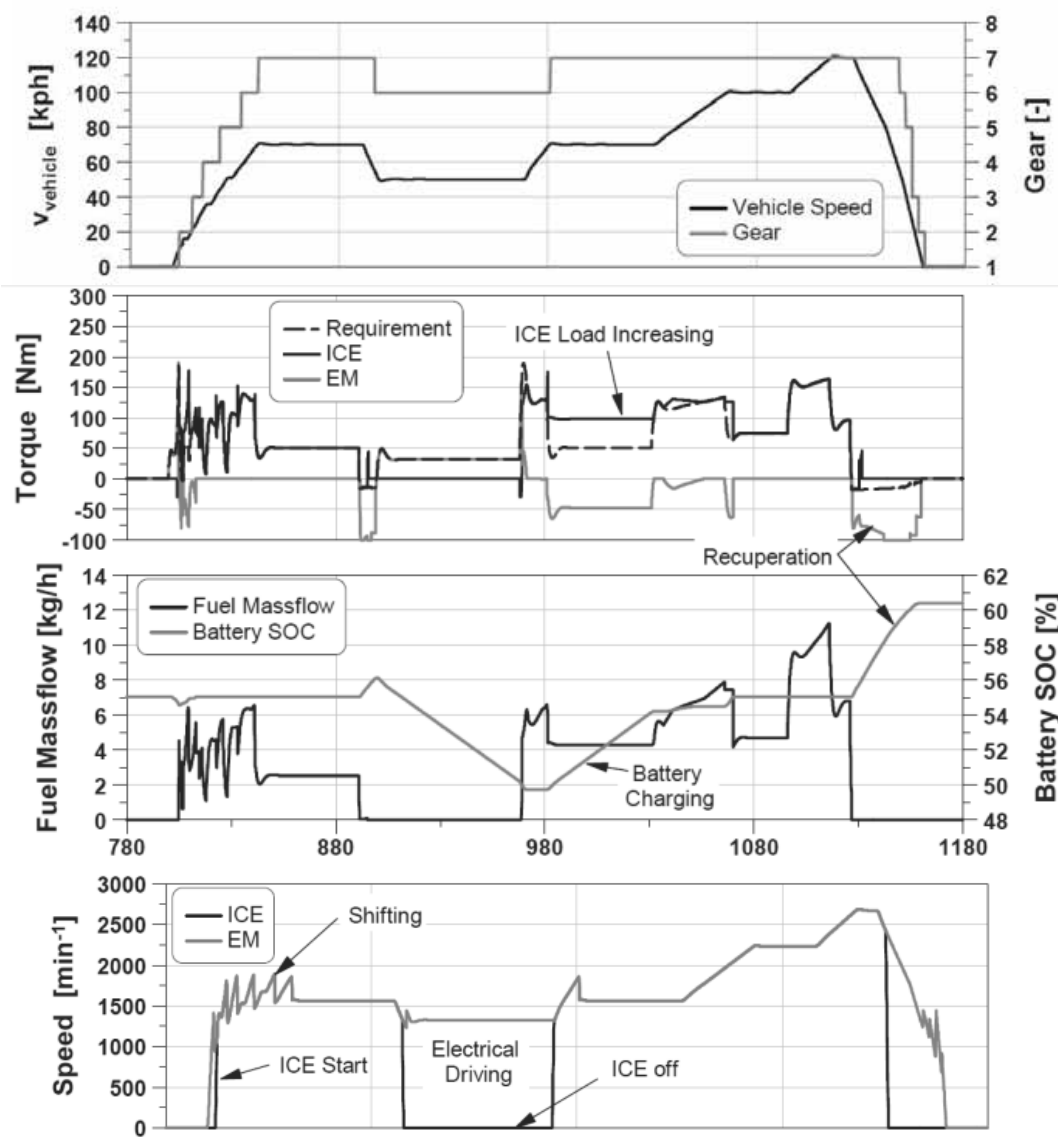

Figure 7. Example of a co-simulated test bench measurement.

\begin{tabular}{|ll|ll|}
$\mathrm{A}$ & vehicle frontal area $\left(\mathrm{m}^{2}\right)$ & $\mathrm{SOC}$ & state of charge $(\%)$ \\
$\mathrm{c}_{\mathrm{w}}$ & drag coefficient $(-)$ & $\mathrm{T}$ & torque $(\mathrm{Nm})$ \\
$\mathrm{E}_{\text {bat }}$ & energy content $(\mathrm{Wh})$ & $\mathrm{X}_{\text {veh }}$ & vehicle distance $(\mathrm{m})$ \\
$\mathrm{F}_{\mathrm{acc}}$ & acceleration resistance $(\mathrm{N})$ & $\dot{x}_{\text {veh }}$ & vehicle speed $(\mathrm{m} / \mathrm{s})$ \\
$\mathrm{F}_{\text {air }}$ & air resistance $(\mathrm{N})$ & $\ddot{x}_{\text {veh }}$ & vehicle acceleration $\left(\mathrm{m} / \mathrm{s}^{2}\right)$ \\
$\mathrm{F}_{\text {bra }}$ & braking force $(\mathrm{N})$ & & traction force $(\mathrm{N})$ \\
$\mathrm{F}_{\text {gra }}$ & gradient resistance $(\mathrm{N})$ & & \\
$\mathrm{f}_{\mathrm{r}}$ & rolling resistant coefficient $(-)$ & $\alpha$ & climbing angle $\left({ }^{\circ}\right)$ \\
$\mathrm{F}_{\text {rol }}$ & rolling resistance $(\mathrm{N})$ & $\eta$ & efficiency $(\%)$ \\
$\mathrm{g}$ & acceleration due to gravity $\left(\mathrm{m} / \mathrm{s}^{2}\right)$ & $\lambda$ & rotating mass coefficient $(-)$ \\
$\mathrm{m}_{\text {veh }}$ & vehicle mass $(\mathrm{kg})$ & $\varrho$ & air density $\left(\mathrm{kg} / \mathrm{m}^{3}\right)$ \\
$\mathrm{P}_{\text {bat }}$ & battery power $(\mathrm{W})$ & & \\
$\mathrm{P}_{\text {veh }}$ & road resistance power $(\mathrm{W})$ & & \\
\hline
\end{tabular}

Nomenclature

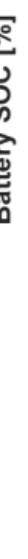

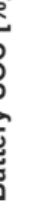

